\title{
LETTERS
}

\section{Radiation Protection in Cardiac Catheterization Laboratory}

Radiation is a part of X ray energy which is an essential pre requisite of imaging. This is an integral part of the cardiac catheterization laboratories. In spite of advances in reducing the doses of radiation in the new generation machines, its effects on the human system cannot be eliminated. The cath lab is a closed atmosphere where the working staff (i.e., cardiologists, cardiac technicians, radiographers, nurses and trainees) is at a potential risk to radiation exposure almost on a daily basis. ${ }^{1,2}$

Compared to other departments (radiology, urology, operating rooms, etc.) that also use x-ray equipment, the cardiac cath lab is generally considered an area where exposure to radiation is particularly high. Factors such as the configuration of the of the x-ray equipment, the number of cases per day, and the often long period of screening required for a study, contribute to this relatively high level of exposure and monitoring results for staff members in the cath lab who wear single badges at the collar outside their lead aprons are generally amongst the highest in the hospital.

Unfortunately ionizing radiation is a workplace hazard that cannot be detected by the human senses. Exposure rates exceeding $7.14 \mathrm{~Gy} / \mathrm{hr}$ (i.e., 5 sievert/hr) in the cath lab have been reported ${ }^{3}$ and interventional procedures such as percutaneous coronary intervention (PCI) and electrophysiological studies (EPS)/ pacing result in the highest radiation exposure to patients and staff.

Radiation in the cath lab is generated using two different modes: fluoroscopy or cine angiography (cine). Fluoroscopy is used for catheter placement and involves 95\% of the total x-ray operation time but only causes $40 \%$ of the total radiation exposure to staff and patients. This is due to pulsed screening that reduces exposure dose. Cine is used to acquire diagnostic images and to generate a permanent record

\section{Correspondence:}

Rehana Kousar

Staff Nurse, Cardiac Cath Lab, Sher-i-Kashmir Institute of

Medical Sciences, Soura Srinagar, 190011

E-mail: kouser971@gmail.com of the procedure and, although representing only $5 \%$ of the total $\mathrm{x}$-ray tube operation time, $60 \%$ of the total radiation exposure to staff and patients occur during cine. This is primarily due to use of relatively high dose rapid sequence screening required to record onto film. Significant reductions in exposure can be realized by being aware of when cine will be used and applying radiation safety measures accordingly.

The average dose per procedure for the cardiologist is estimated as $0.05 \mathrm{mGy}$. To allow better comparison of patient and staff doses this value can be expressed as the dose area product (DAP). The DAP is calculated as the product of dose in air in a given plane and the area of the irradiating beam and is independent of the distance from the $\mathrm{x}$-ray source. Coronary angiography and PCI produce mean-patient DAPs in the range 20 to $106 \mathrm{~Gy} . \mathrm{cm}^{2}$ and 44 to $143 \mathrm{~Gy} . \mathrm{cm}^{2}$ respectively. $^{3}$

\section{Potential Hazards of Radiation}

Injury to skin: Prolonged exposure can lead to erythema , necrosis and non healing ulcers. Typically 1 minute of screening leads to an exposure of $20 \mathrm{mGy}$ skin dose. Threshold for transient skin erythema is $2 \mathrm{~Gy}$. Prolonged and sustained exposure can lead to altered cellular DNA responsible for skin cancers.

Damage to Eyes: Higher doses of radiation can damage sclera, conjuctiva, iris and blood vessels of retina. Lens is very susceptible to get damaged irreversibly leading to premature cataracts. These cataracts involve posterior part of the lens.

Cancers: The small probability of damaging the multiplying cells and changing their growth pattern can lead to malignancies. The incidence of these cancers increases with the degree and length of exposures. The organs at highest risk of developing these cancers are brain, thyroid and skin. Gonads are at a lower risk of developing cancers.

Gy. unit

The incremental fatal cancer risk is estimated at $4 \%$ per

Genetic effects: Genetic effects caused by radiation have also been demonstrated in animal models. However genetic 
effects are yet to be observed in humans, even when exposed to relatively high levels of radiation.

\section{Pregnancy and Risk to fetus}

As a general rule, the sensitivity of a tissue to radiation is directly proportional to its rate of proliferation. Therefore, one could infer that the human fetus, because of its rapid progression from a single cell to a formed organism in nine months, is more sensitive to radiation than the adult .This inference is supported by the results of experiments in animal models, and the experience with human populations that have been exposed to very high doses of radiation (atomic bombing victims). In humans, the major deleterious effects on the fetus include fetal wastage (miscarriage), teratogenicity (birth defects), mental retardation, intrauterine growth retardation and the induction of cancers such as leukaemia that appear in childhood. Birth defects and mental retardation are the adverse effects that are of the most immediate concern for the expectant mothers. Fortunately, not all exposures to ionizing radiation result in these outcomes. The risk to the fetus is a function of both gestational age at exposure and the radiation exposure.

The threshold for childhood cancer induction is not clear cut. Despite these uncertainties in the dose-effect relationship, some broad generalizations based on fetal dose ranges may be made.

Fetal dose less than 0.01 Gy unit: There is no evidence supporting the increased incidence of any deleterious developmental effects on the fetus. Congenital malformations, mental retardation, intrauterine growth retardation and childhood cancer is believed to be low compared to the baseline risk.

Fetal dose exceeding 0.1 Gy unit: The lower limits for threshold doses for effects such as mental retardation and diminished IQ and school performance fall within this range.

\section{How to Reduce Radiation Exposure? \\ Reducing the time of exposures:}

Average time taken for carrying out a coronary angiography varies between 10 to 20 minutes and for carrying out a PCI procedure 45 to 90 minutes depending on the complexity of the procedure. The time utilized for fluoroscopy and cine angiography during these procedures however is very variable depending on the experience of the operator. The lower the amount of time spent in a radiation area, the lower the exposure will be. Significant reductions can be achieved when an activity is delayed until after cine imaging is completed. Every effort should be made by the operating cardiologist in the cath lab to minimize fluoroscopy and cine screening time. ${ }^{4-6}$

Distance from the beam and angles: Increasing the distance from the radiation beam decreases the exposure. Doubling the distance decreases the radiation exposure by 4 times. The angle from which the camera is exposed also has important implications. Left oblique views especially the anterior (LAO) give much more radiation as compared to the right oblique views which are directed away from the operators.
Likewise the cranial views give much higher exposures than the caudal projections. It is therefore advisable to take only the necessary views for a particular case depending upon the need rather than all the possible projections.

In general the first operator gets more radiation than the second operator or the assistant. Persons whose presence is not essential during the procedure should be discouraged from being around the imaging equipment.

\section{Shielding}

Lead shields and shielding will significantly reduce the risk of exposure but only if appropriately used and in proper working order. Protective equipment includes lead aprons, thyroid collars and leaded glasses. With the newly designed frames and ultra light lenses, protective leaded eyewear is now used by more of the cardiologists and staff in cardiac cath lab. Some cath labs also use overhanging lead screens to prevent radiation exposure to brain. The staff should wear a protective apron of at least $0.25 \mathrm{~mm}$ lead equivalent. Protective gloves should be of at least $0.35 \mathrm{~mm}$ lead equivalent. All such protective clothing should bear an identifying mark and should be examined at yearly intervals. Defective items should be withdrawn from use.,

\section{Periodic calibration and maintenance of the equipment}

The following requirements are necessary and are undertaken by trained personnel periodically.

Adequate total filtration is present.

The fluoroscopy timer terminates the exposure or produces an audible signal at the end of a five-minute accumulative time interval.

During fluoroscopy, x-ray field collimation and alignment with the image intensifier is appropriate.

Fluoroscopic exposure rates do not exceed the regulatory standards.

Patient exposure information has been obtained for the simulated clinical conditions and is posted where it is readily available to the physician during the fluoroscopic procedure.

\section{Guidelines and Protocols}

Every institution should have working protocols set up to minimize radiation exposure and enumerate the protection strategies. These must be read, understood and strictly adhered to in everyday practice. Staff must comply with these local rules in order to ensure that the Trust and all their employees do not contravene statutory requirements of the ionizing radiation regulations and other relevant legislation.

The guidelines can be drawn from several documents on this subject and are readily available. American and European guidelines as also directives from Bhabha Atomic Research center can be made as reference points.

\section{References}

1. Hart D, Jones DC and Wall BF. Estimation of effective dose in diagnostic radiology from enterance surface 
dose and dose area product measurements, National Radiological Protection Board (NRBP-R 262),HMSO publications centre, London pp 1-57, 1994.

2. Leung $\mathrm{KC}$ and Martin CJ. Effective doses for coronary angiography. British Journal of Radiology 1996;69: 426-31.

3. Bakalyar MD, Castellani MD and Saffian RD. Radiation exposure to patients undergoing diagnostic and interventional cardiac catheterization procedures. Catheterization and Cardiovascular Diagnosis 1997);42: 121-25 (Comments, pp 126-129).
4. Betson S, Efstathopoulos EP, Katritis D, et al. Patient radiation doses during cardiac catheterization procedures. British Journal of Radiology 1998:71;634-39.

5. Katritsis D, Efstathopoulos E, Betsou S, et al. Radiation exposure of patients and coronary arteries in the stent era: A prospective study. Catheter Cardiovasc Interv 2000;51:259-64.

6. Kuon E, Glaser C, Dahm JB. Effective techniques for reduction of radiation dosage to patients undergoing invasive cardiac procedures. BrJ Radiol 2003;76:406-13.

Rehana Kouser, RN

Cardiac Catheterization Laboratory,

Sher-i-Kashmir Institute of Medical Sciences, Soura Srinagar, Kashmir. 